\title{
Experimental Factors Affecting the Quality and Reproducibility of MALDI TOF \\ Mass Spectra Obtained from Whole Bacteria Cells
}

\author{
Tracie L. Williams, Denis Andrzejewski, Jackson O. Lay, Jr., \\ and Steven M. Musser \\ Instrumentation and Biophysics Branch, Center for Food Safety and Applied Nutrition, United States Food \\ and Drug Administration, College Park, Maryland, USA
}

\begin{abstract}
Numerous experimental factors are shown to significantly influence the spectra obtained when bacteria are analyzed by MALDI TOF/MS. Detailed investigation of the instrument parameters and sample preparation are all shown to influence the spectra. Of these, the preanalysis sample preparation steps incorporate the most important elements influencing the quality and reproducibility of the spectra. Some of the most important sample preparation factors include the method employed for sterilization, the type of matrix, the matrix solvent and concentration of cells in the matrix, as well as the type and concentration of acid added to the matrix. The effects of these parameters, as well as other aspects of sample preparation and the effects of several instrumental parameters on spectra are presented. Optimization and control of all experimental variables leads to a stable protocol for analysis of bacteria. The protocol employs a Nd:Yag laser and describes both sample handling and instrument conditions which consistently yield reproducible MALDI TOF mass spectra with greater than 25 peaks from both gram-positive and gram-negative bacteria. (J Am Soc Mass Spectrom 2003, 14, 342-351) (c) 2003 American Society for Mass Spectrometry
\end{abstract}

$\mathrm{M}$ atrix assisted laser desorption/ionization time-of-flight mass spectrometry (MALDI TOF/MS) has been used routinely for the analysis of purified proteins, including proteins isolated from bacteria [1]. Recently, the technique was introduced as a new method for directly and rapidly identifying bacteria based on the simultaneous detection of proteins from "whole" cells, even in the presence of other cellular components [2-4]. Identification was based upon the complex patterns observed and the characteristic nature of bacterial mass spectra. Even in the earliest studies, differences in spectra were noted at both the genus and the species level. While the spectra from "whole cells" (no separation of cellular components) reflect only a small portion of the cellular proteome, they are nevertheless sufficiently characteristic to address many taxonomic or biological questions. Recently, it has also been demonstrated that simple fractionation of cellular components using membranes greatly increases the total number of proteins detected

Published online March 10, 2003

Address reprint requests to Dr. S. M. Musser, Instrumentation and Biophysics Branch, Center for Food Safety and Applied Nutrition, US Food and Drug Administration, 5100 Paint Branch Pkwy. HFS-717, College Park, MD 20740, USA.T. L.S. M. E-mail: steven.musser@cfsan.fda.gov in all of the fractions, presumably because of a reduction of ion-suppression effects from mixtures with fewer components [5]. While these additional steps may sometimes be necessary, often they are not [6]. Moreover, the simplicity of the methodology and the rapidity of the method are attractive features of the "whole-cell" approach.

Among a number of important potential applications of this technique, several are related to public health and food safety. Based on a report published in 2000 by the Centers for Disease Control and Prevention, there were over 76,000,000 food-borne illnesses in the United States in 1999 [7]. This included 325,000 reported hospitalizations and 5000 deaths as a direct result of microbial contamination of food. While problems with such organisms as Lysteria monocytogenes, Escherichia coli, and Salmonela enteritidis are well known, the vast majority of the illnesses and $64 \%$ of the deaths were from unknown organisms [7]. Clearly, methods are needed for rapid screening and identification of both expected and unexpected microorganisms in the food supply. This is even more important in light of the emergence of pathogenic microorganisms such as Escherichia coli H7:O157. Although a number of biochemical or molecular biological methods such as ELISA and 
PCR have been developed for the rapid detection of these specific organisms, additional methods are needed which have the capability of differentiating pathogenic strains as well as identifying biomarkers of pathogenicity and virulence. Several important public health questions remain unanswered because the existing methods are either inadequate or inappropriate for these purposes. MALDI TOF/MS might be applied to the solution of some outstanding bacterial food-safety problems based on the detection of either biomarkers for specific bacterial properties, such as antibiotic resistance, or on the identification of specific strains of organisms. Moreover, while both the PCR and MALDI TOF/MS techniques generally rely on preanalysis culture steps, the detection of biomarker proteins directly from contaminated water, lettuce, and cloth by MALDI $\mathrm{TOF} / \mathrm{MS}$, without resorting to preanalysis culture steps was recently reported [8].

Three general approaches involving MALDI mass spectrometry and bacterial characterization have been developed. One concerns the detection of specific biomarker proteins indicative of specific genotypic or phenotypic properties [9]. The second approach utilizes protein database searches to rank or identify organisms $[10,11]$. The third approach employs spectral comparisons designed either to identify or rule out specific species or strains [2-4]. While all of these approaches require some level of reproducibility, the comparison of reference and unknown spectra for bacterial identification (or differentiation) requires the highest level of reproducibility between spectra [12]. For MALDI TOF/MS of bacteria, reproducibility of spectra has been problematic for both methodological and biological reasons [13-15]. Nevertheless, the creation of spectral libraries for the rapid identification of unknown bacteria, using the simple "whole cell" approach remains an important goal for a number of research groups and instrument manufacturers $[10-12,16,17]$.

A recent inter-laboratory study by $\mathrm{Li}$, Long, and coworkers suggests that a complex procedure involving cell washes, lyophylization, vortexing, centrifugation, and a two-layer matrix/sample deposition approach can yield reproducible spectra [18]. Most of the "whole cell" studies to date have been conducted using a nitrogen laser. We wish to report on results using a Nd:Yag laser with a protocol that is fast, simple, and yields quality spectra with good resolution and spectral information. The procedure retains the simplicity of the whole-cell method and can be used on a variety of bacteria with good reproducibility. The method is demonstrated using Listeria innocua and Escherichia coli bacteria. These organisms were chosen as model organisms since members of their respective species are important to food safety and they represent both gram-positive and gram-negative organisms. A number of parameters that influence spectral behavior using this protocol are discussed.

\section{Experimental Conditions}

\section{Materials}

Sodium chloride was purchased from Fisher Scientific Co. (Fairlawn, NJ). Ethanol and acetonitrile were purchased from Burdick and Jackson Co. (Muskegon, MI). Formic acid (FA), trifluoroacetic acid (TFA), phenol, formaldehyde, $\quad \alpha$-cyano-4-hydroxycinnamic acid (CHCA), 3,5-dimethoxy-4-hydroxycinnamic acid (sinapinic acid), 4-hydroxy-3-methoxycinnamic acid (ferulic acid), Triton X-100, Tween 20R, polyoxyethylene-8lauryl ether, $\mathrm{n}$-dodecyl $\beta$-D-glucopyranoside, octyl $\beta$-Dglucopyranoside, and octyl $\beta$-D-thioglucopyranoside were purchased from Sigma-Aldrich Chemical Co. (St. Louis, MO.). All chemicals were technical grade or better and were used as received except CHCA. CHCA was recrystallized following the sample preparation procedure in the TofSpec E operator's manual. Briefly, CHCA $(100 \mathrm{mg})$ was dissolved in $10 \mathrm{~mL}$ of water and ammonium hydroxide added until almost all the matrix had dissolved. Concentrated $\mathrm{HCl}$ was added slowly until a large amount of matrix had precipitated $(\mathrm{pH}$ $\sim 2$ ). The precipitate was filtered, washed several times with $0.1 \mathrm{M} \mathrm{HCl}$ and dried overnight in a vacuum desiccator.

Micro-spin centrifuge filters, 0.45 um polyvinyldene difluoride (PVDF), were purchased from Alltech Associates Inc. (Deerfield, IL). Nano-Spin membrane filters $10 \mathrm{~K}$ molecular weight cutoff (MWCO) were purchased from Gelman Sciences, (Ann Arbor, MI).

The organisms used in this study, Escherichia coli (gram-negative, no. 1224) and Listeria innocua (grampositive, no. 1240) were obtained from reference cultures maintained at the FDA's Center for Food Safety and Applied Nutrition, College Park, MD.

\section{Methods}

Cells were grown for $24 \mathrm{~h}$ on tryptic soy using either agar plates or liquid culture methods. Cells grown by liquid culture were grown in $50 \mathrm{~mL}$ of tryptic soy broth plus yeast extract at $35^{\circ} \mathrm{C}$. Faster growing cells (E. coli) were reinoculated once and grown under aeration to get mid-log phase cells whereas the slower growing cells (Listeria) were used directly after the initial inoculation. Cells (at a concentration of approximately 1.0 $\mathrm{OD}_{610} / 1 \mathrm{~mL}$ ) were harvested by centrifugation at 12,000 $\mathrm{rpm}$ for $2 \mathrm{~min}$. The broth was removed by aspiration, 1 $\mathrm{mL}$ of a sterilization solution was added directly to the cells, and the solution was vortexed for $1 \mathrm{~min}$. Alternatively, cells grown on plates were removed by scraping and added directly to $1 \mathrm{~mL}$ of a sterilizing solution to give a final concentration of approximately $1.0 \mathrm{OD}_{610}$. Solutions used for sterilization were sterilized distilled water (with boiling), 5\% phenol, $8 \%$ formaldehyde in $70 \%$ ethanol, $70 \%$ ethanol, and a lysozyme solution. Suspensions of "unsterilized" cells were also prepared using either sterilized distilled water or a $0.9 \%$ sodium 
chloride solution. The suspensions of bacteria were transported to the mass spectrometry laboratory where they were centrifuged at $4000 \mathrm{rpm}$ for $4 \mathrm{~min}$ and the bulk $(0.6 \mathrm{~mL})$ of the solution removed. The remaining (approximately $400 \mu \mathrm{L}$ ) suspension was analyzed immediately. The bacteria samples were stored in a refrigerator at $4{ }^{\circ} \mathrm{C}$ when not in use.

At the time of analysis, the cells were resuspended in the remaining solution by agitation. Approximately 2 $\mu \mathrm{L}$ of this bacterial suspension was mixed directly with the matrix solution at ratios of 1:1, 1:3, and 1:5. The matrix compounds used to produce matrix solutions were CHCA, sinapinic acid, and ferulic acid. CHCA (10 $\mathrm{mg}$ ) or sinipinic acid $(10 \mathrm{mg})$ were dissolved in $1 \mathrm{~mL}$ of a suitable solvent (50/50 ethanol/water, 50/50 methanol/water, 50/50 acetonitrile/water, 50/50 tetrahydrofuran (THF)/water, or 50/50 acetone/water) containing between $0.1-10 \%$ TFA or FA. In some experiments a small amount of surfactant $(0.1-1 \%$ of Tween 20R, Triton X-100, polyoxyethylene-8-lauryl ether, n-dodecyl $\beta$-D-glucopyranoside, octyl $\beta$-glucopyranoside, or octyl $\beta$-D-thioglucopyranoside) was also added to the matrix solution. Ferulic acid crystals $(12.5 \mathrm{mg})$ were dissolved in $1 \mathrm{~mL}$ of reagent comprised of $17 \%$ formic acid, $33 \%$ acetonitrile, $50 \% \mathrm{H}_{2} \mathrm{O}$. Two $\mu \mathrm{L}$ of the sample and matrix mix was spotted onto a 96 well stainless steel plate and allowed to air dry (dried-droplet method). In experiments where the bacterial suspension was filtered using a micro-spin centrifuge filter, the matrix solution was added and mixed directly with the cells remaining on the filter after a $200 \mu \mathrm{L}$ aqueous wash. The sample and matrix mix was then transferred to the sample plate. The two-layer method of depositing the sample was also tried by placing a thin layer of matrix on the plate prior to sample/matrix deposition. This method provided no advantages over the dried-droplet method in the detection of proteins when using the Nd:Yag laser. For experiments where the conditions are not specified, the matrix was CHCA in 50/50 acetonitrile/water containing $2.5 \%$ TFA.

\section{Instrumentation}

MALDI spectra were recorded on a Micromass TofSpec 2E time-of-flight mass spectrometer operated in the positive ion, linear mode. The instrument was fitted with both a Minilite II Nd:Yag laser (Continuum, Santa Clara, CA) and a nitrogen laser (standard with the instrument). Selection of the laser used for ionization was performed directly through the software and required no adjustments to the individual lasers. Samples were analyzed using an accelerating voltage of $25 \mathrm{kV}$, except where specifically noted. Ionization was achieved using either the Nd:Yag laser at a wavelength of $355 \mathrm{~nm}$ or a nitrogen laser at $337 \mathrm{~nm}$. The laser power at the target was measured while the instrument was vented using an Orion/PE laser power monitor (Ophir Optronics LTD, Peabody, MA). For the Nd:Yag laser, the initial power output needed to observe ions was high, around 70 microjoules. This was dropped to a lower optimum range after instrument/laser conditions equilibrated (about $30 \mathrm{~min}$ ). This type of equilibration was not necessary with the nitrogen laser. The optimum laser power for the $\mathrm{Nd}$ :Yag laser was between 30 and 45 microjoules and for the nitrogen laser between 10 and 25 microjoules. The optimal settings were determined by lowering the laser energy to a point slightly above the desorption and ionization threshold of the sample. The laser was fired at 2 shots per $\mathrm{s}$ and 10 shots accumulated per scan. Each spectrum represents the average of about 15 scans. The data was externally calibrated using a mixture of insulin and myoglobin.

\section{Results and Discussion}

\section{Growth and Sterilization of Bacteria}

Bacterial growth. MALDI spectra from bacteria grown in tryptic soy on either traditional agar plates or in liquid culture media were compared as variables were changed during the experimental procedures listed below. In the course of these studies, no obvious effects were observed that could be attributed to spectral differences associated with the use of either liquid culture or traditional plate-culture methods for either $E$. coli or Listeria. The method variables that were evaluated appeared to affect the quality (i.e., resolution, number of peaks, intensity, and mass range of proteins detected) of the bacteria spectra in a similar way regardless of how the same species of bacteria was grown. This result was verified using the final set of experimental conditions described in this work as the "optimum" method for MALDI TOF/MS analysis of whole cells sterilized with $70 \%$ ethanol. It is important to note however, that growth time will affect reproducibility of spectra. This factor has been confirmed by several groups and was therefore strictly adhered to for these studies $[5,17]$.

Bacterial sterilization. Although gamma irradiation has been shown to be an effective means for both sterilizing and preserving bacterial samples [20,21], it requires specialized equipment generally unavailable at field collection sites. Thus, we have focused on the use of commonly available sterilization solutions. Our aim was to find a solution which not only provided an effective means for killing the bacteria, but also produced samples which could be safely transported and easily stored prior to analysis, while giving reproducible spectra with many proteins detected when analyzed immediately or later after transportation and storage. Using Listeria as a model organism, substantial differences were observed in the mass spectra of bacteria, depending upon the composition of the solution used for sterilization. The variability spanned the range from little or no observable proteins for sterilization using formaldehyde, phenol or lysozyme, (data not shown) to numerous proteins in cells boiled in sterilized 
(a)

(b)

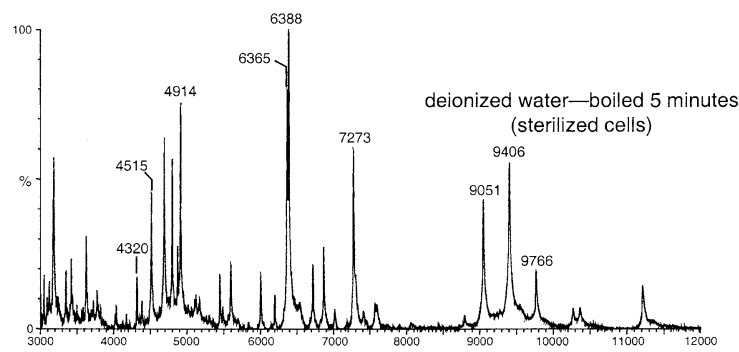

(c)

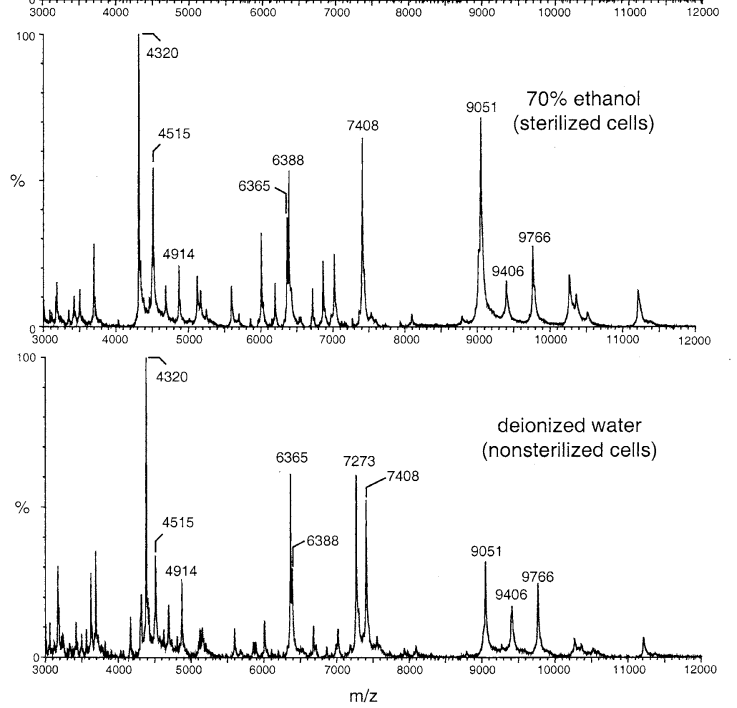

Figure 1. The MALDI mass spectrum of Listeria innocua cells after (a) sterilization via boiling for $5 \mathrm{~min}$., (b) sterilization with $70 \%$ ethanol, and (c) no sterilization.

distilled water for 2-5 min (Figure 1a) or treated with $70 \%$ ethanol (Figure 1b). We attribute the lack of protein-like ions from cells treated with phenol and formaldehyde to the same mechanisms by which they exhibit their antibacterial activity. Formaldehyde, an excellent cell-killing agent, forms permanent covalent bonds with cellular proteins whereas phenol, a less efficient antibacterial, reversibly precipitates proteins within the cell [22]. Similar results were obtained with E. coli (data not shown).

Non-sterilized Listeria cells were examined for comparison. Significant signals were observed from the bacteria treated with either deionized water (Figure 1c) or a solution of $0.9 \%$ sodium chloride. The spectra showed peaks corresponding to a subset of the same ions seen from suspensions of the ethanol-treated cells, although the spectra obtained from cells treated with $0.9 \% \mathrm{NaCl}$ was of poorer quality.

Attempts to increase the number of proteins detected by the removal of salts and other non-protein related material from the sodium chloride, lysozyme and phenol sterilized samples by washing the sample $(200 \mu \mathrm{L}$ aqueous wash of the bacterial suspension through a $0.45 \mu \mathrm{m}$ PVDF micro-spin centrifuge filter) typically did little to improve spectra for any of the bacterial suspensions. In many cases, either the spectra were nearly identical regardless of whether or not the suspensions were washed to remove salts or there was a slight reduction in signal for proteins below $10 \mathrm{kDa}$ (data not shown). However, one exception was noted: While no protein-like peaks were observed from a phenol suspension of cells, numerous protein-like peaks were observed after a $200 \mu \mathrm{L}$ aqueous wash that presumably removed phenol along with any salts.

Cells boiled in water or suspended in $70 \%$ ethanol produced spectra containing even more protein ion signal than the non-sterilized cells (Figure 1). For the two types of bacteria used in this study, and also for numerous other bacteria analyzed in our laboratory, a large number of peaks corresponding to proteins have always been observed from cells treated with either 70\% ethanol or by boiling. Although the addition of alcohol to whole cells is widely believed to lead to membrane disruption [22, 23], MALDI analysis of the ethanol supernatant does not show leaching of the proteins out of the cells and into solution (data not shown). Furthermore, ethanol sterilization neither precipitates the proteins in such a manner that they cannot be recovered from the cellular debris, nor does it cause covalent bonding that would prohibit detection of proteins. While placing the vial of cells in boiling water for several minutes also produces bacterial suspensions from which many proteins can be observed, excessive heating can sometimes lower the quality of spectra (data not shown). More importantly, boiling water would likely be less accessible for use in field collection of bacteria than $70 \%$ ethanol. In addition, the long-term storage of bacteria in $70 \%$ ethanol is very robust. Bacteria stored in the refrigerator and analyzed weeks after the initial sterilization produced spectra identical to those obtained immediately after sterilization. The effectiveness of ethanol sterilization was checked by reinoculating tryptic soy broth and nutrient agar with $E$. coli and Listeria cells after sterilization in $70 \%$ ethanol for $5 \mathrm{~min}$. No cell growth could be detected in either media after $12 \mathrm{~h}$ of incubation. For these reasons, 70\% ethanol was selected as the method of choice for producing sterile bacterial suspensions for MALDI analysis. Although this method of sterilization was effective for the organisms used in this study, it is important to take appropriate precautions when working with bacteria. Certain types of bacteria may not be killed in 70\% ethanol. This would be true for any type of bacteria that can produce spores. A summary of the results observed using the different sterilization solutions for bacteria suspension are summarized in Table 1 . For the experiments listed in Table 1, no differences were observed between cells from liquid cultures and the cells collected from agar plates.

\section{Matrix Effects and Matrix Additives}

Matrix compounds. CHCA was the matrix used to determine that $70 \%$ ethanol represents a practical and effective method for producing sterile bacterial suspensions suitable for analysis by MALDI TOF/MS. Because sinapinic acid and ferulic acid have also been reported 
Table 1. A comparison of various suspension solutions for bacterial sterilization and their effects on MALDI TOF Mass spectra of E. coli and Listeria

\begin{tabular}{lccc}
\hline Solution & Sterilization & $\begin{array}{c}\text { MALDI-TOF } \\
\text { Spectra }\end{array}$ & Figure \\
\hline \hline $0.9 \% \mathrm{NaCl}$ & No & Poor & Not shown \\
8\% Formaldehyde & Yes $^{1}$ & None & Not shown \\
$5 \%$ Phenol & No & None & Not shown \\
Lysozyme & Yes & None & Not shown \\
Boiling water & Yes & Good & $1 \mathrm{a}$ \\
70\% Ethanol & Yes & Good & $1 \mathrm{~b}$ \\
Deionized water & No & Good & $1 \mathrm{c}$ \\
\hline
\end{tabular}

${ }^{1}$ Cells were sometimes recultured from formaldehyde suspensions, indicating incomplete sterilization

${ }^{2}$ MALDI spectra were obtained from phenol-treated cellular suspensions after washing with a small volume of water.

$[5,15,19]$ to be useful for the analysis of bacterial cells, especially in the detection of higher mass proteins or peptides, all three were compared for use as matrix compounds with the $\mathrm{Nd}$ :Yag laser for the analysis of bacteria sterilized via $70 \%$ ethanol.

E. coli samples were analyzed using CHCA or sinapinic acid dissolved in 50/50 acetonitrile/water. The $\mathrm{pH}$ of the matrix solution was adjusted by addition of either 1.0 or $2.5 \%$ of TFA or FA to the matrix solution. While both matrix compounds produced spectra with a number of significant peaks between 2 and $12 \mathrm{kDa}$, at comparable acid concentrations, the addition of TFA always produced a larger number of intense peaks than did FA. This is clearly indicated by the MALDI spectra of $E$. coli shown in Figure 2a and b in which sinapinic acid was used as the matrix. Significantly fewer proteins were observed for the same approximate cell concentration with $1.0 \%$ FA than with $1 \%$ TFA and the signal was weaker. Figure 2c shows the spectrum of the same bacteria run in CHCA with $1.0 \%$ TFA. Very similar spectra were observed for both sinapinic acid and CHCA with 1\% TFA. The sinapinic acid matrix sometimes enhanced signals that were less intense using CHCA, but this trend was generally not observed above a mass of $10 \mathrm{kDa}$ and was very difficult to reproduce. CHCA was better for the detection of proteins between 12 and $25 \mathrm{kDa}$ than sinapinic acid, although the masses in this range were not very intense with either matrix. Attempts to enhance the high mass response by either washing the sample to remove salts using micro-spin centrifuge filters or by using a $10 \mathrm{~K}$ molecular weight cutoff selective centrifugal concentrator resulted in only a marginal increase in the detection of higher mass proteins. However, this slight enhancement may simply be the result of alleviating ion suppression caused by low mass proteins as these proteins are effectively removed in the sample wash. Additionally, CHCA produced a more homogeneous sample/ matrix spot on the target. The results of this observation meant that the laser could be focused anywhere on the target when CHCA was used while sinapinic acid often produced "hot spots" and required searching for an

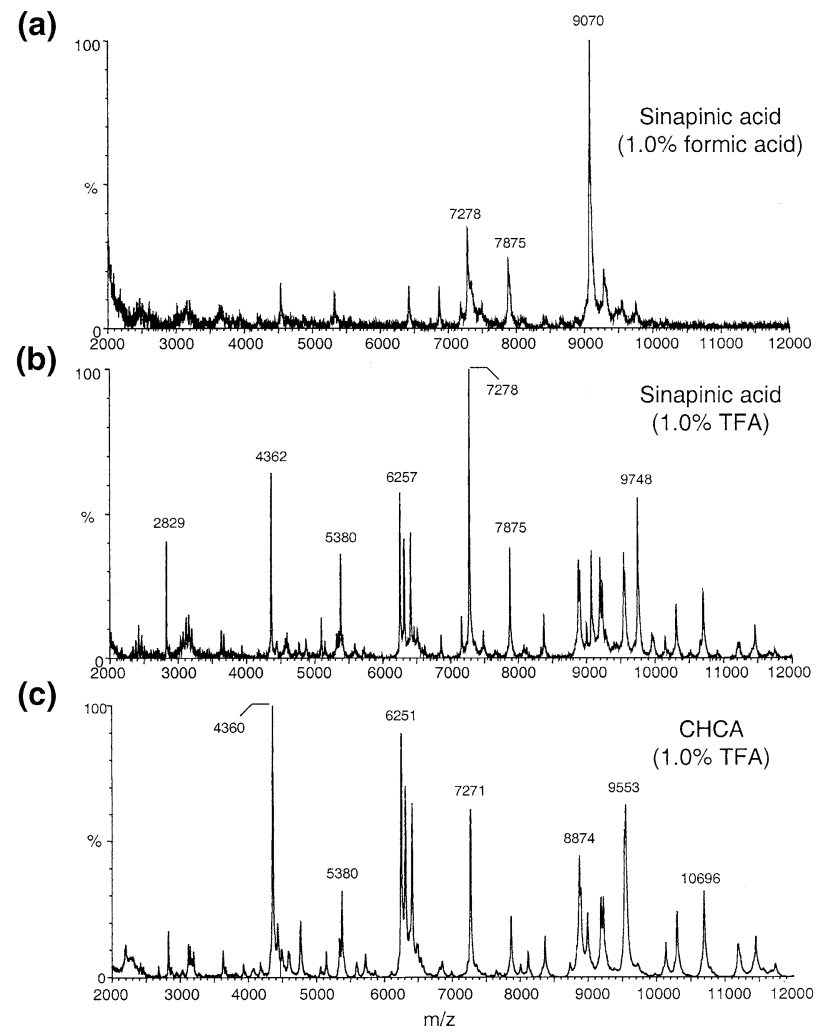

Figure 2. The spectra of E. coli cells using (a) sinapinic acid matrix in $1 \%$ formic acid, (b) sinapinic acid in 1\% TFA, and (c) CHCA in $1 \%$ TFA.

optimum spot to extract data. The same series of experiments described above were repeated using Listeria with similar results observed.

A number of experiments were conducted using ferulic acid as the sample matrix. Voorhees and coworkers reported [19] that ferulic acid in combination with $17 \%$ formic acid gave optimum results for the production of higher molecular weight proteins from whole cells using a nitrogen laser. This prompted us to investigate its effect on bacteria using the $\mathrm{Nd}$ :Yag laser. Listeria was examined using conditions identical to those reported by Voorhees. Some high mass ions were obtained but most of the signal was carried in several ion clusters below $12 \mathrm{kDa}$ (Figure 3a). This spectrum was not easily obtained. The shot-to-shot spectral reproducibility was poor and we obtained only sporadic bursts of signal across the target sample. For comparison, the spectrum of Listeria in CHCA displayed over the same mass range is shown in Figure $3 \mathrm{~b}$. A substantially greater number of protein signals between 3 and $12 \mathrm{kDa}$ were obtained using CHCA than ferulic acid. Also, the signal with ferulic acid was an order of magnitude lower than with CHCA. Similar results were obtained for bacteria mixed with ferulic acid and analyzed using the nitrogen laser. We attribute the differences between our results and those of Voorhees and coworkers to be related to differences in the MALDI TOF instruments used, an example being the distance 

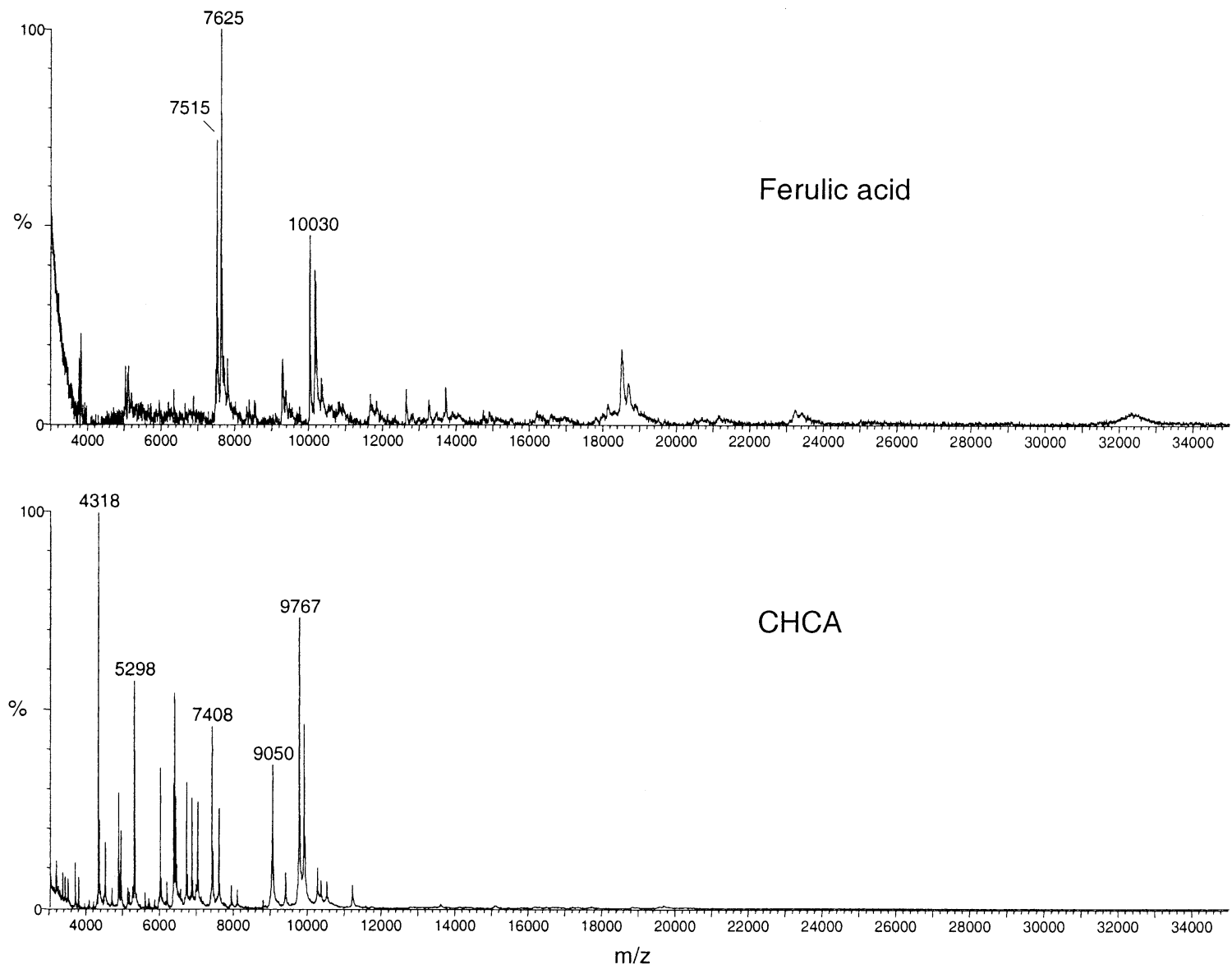

Figure 3. MALDI spectrum of Listeria using the Nd:Yag laser (a) ferulic acid matrix and (b) CHCA matrix.

between the sample plate and the extraction grid. This distance is much smaller on a Voyager-DE STR than on the Micromass TofSpec 2E.

Based on the experiments described above, CHCA was selected as the optimum matrix for analyzing whole bacteria cells sterilized with $70 \%$ ethanol.

Acid concentration in matrix. Because of concerns that the matrix solvent and the acid used for $\mathrm{pH}$ modification might affect the spectra from bacterial cells, these parameters were investigated separately. The effects of the acid used to adjust the $\mathrm{pH}$ of the CHCA matrix solution were examined. For E. coli cells treated with $70 \%$ ethanol, a 50/50 acetonitrile/water matrix solution containing variable amounts of TFA or FA $(0.1,0.5,1$, $2.5,5$, or $10 \%$ ) was analyzed by MALDI to evaluate the effects of different acid concentrations on the spectra of bacteria. Unlike the previously described experiment using sinapinic acid in which no significant signals were observed using FA concentrations of 0.1 and $0.5 \%$, proteins were detected for CHCA with the addition of only $0.1 \%$ FA (Figure $4 a$ ). The number and intensity of proteins increased significantly as the concentration of FA went to 5 and $10 \%$. When modifying the CHCA matrix $\mathrm{pH}$ with TFA the spectral signal intensity rose more quickly with increasing acid concentration than with FA and began to drop off at concentrations greater than 3\% TFA (Figure 5). These observations are consistent with the better ion pairing ability of TFA and its enhanced ability to solubilize the proteins in the matrix. For TFA, the optimum detection of small to medium size proteins ( 3 to $20 \mathrm{kDa}$ ) was between 1 and $2.5 \%$. Above this concentration the signal of the higher mass proteins began to disappear. This is probably due to multiple charging of the proteins occurring at the higher acid concentrations. Based on these experiments, we concluded that the optimum acid concentration when using CHCA was about $2.5 \%$ TFA.

Matrix solvent composition. It was of interest to investigate the effect of the various solvent matrix mixtures on the detection of cell proteins. The solvent can affect the MALDI spectra by its direct interaction with the bacteria cells as well as with how well it incorporates the bacteria with the matrix in the crystallization process. If poor crystallization occurs the ionization process may not be efficient. Ethanol sterilized E. coli and Listeria suspensions were analyzed using CHCA dissolved in the following solutions: 50/50 methanol/ water, 50/50 acetone/water, 50/50 THF/water, or 
(a)

(b)
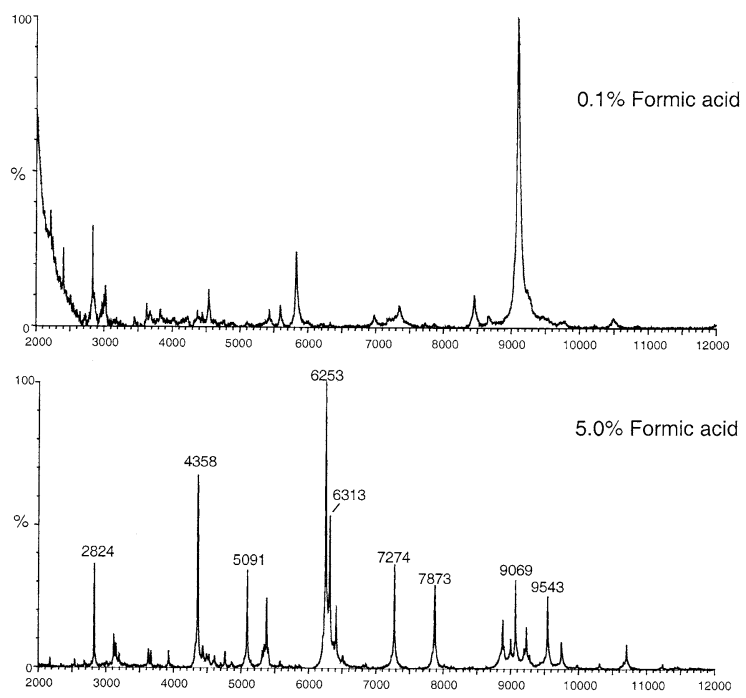

(c)

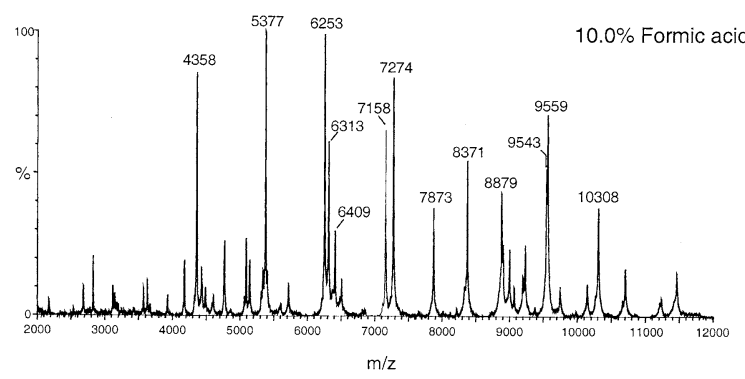

Figure 4. The effect of formic acid concentration on the number of protein peaks observed in the MALDI spectrum of E. coli in CHCA, (a) $0.1 \%$ FA, (b) 5\% FA, and (c) 10\% FA.

50/50 acetonitrile/water (each with 2.5\% TFA). Both species of bacteria behaved similarly in the various solvent systems. Use of different solvents did not result in the detection of markedly different proteins. The main difference was in the number and intensity of the proteins observed. The CHCA dissolved in methanol or THF resulted in spectra that looked very similar. Compared to the other solvent/matrix mixtures they produced spectra with the least number of proteins (Figure

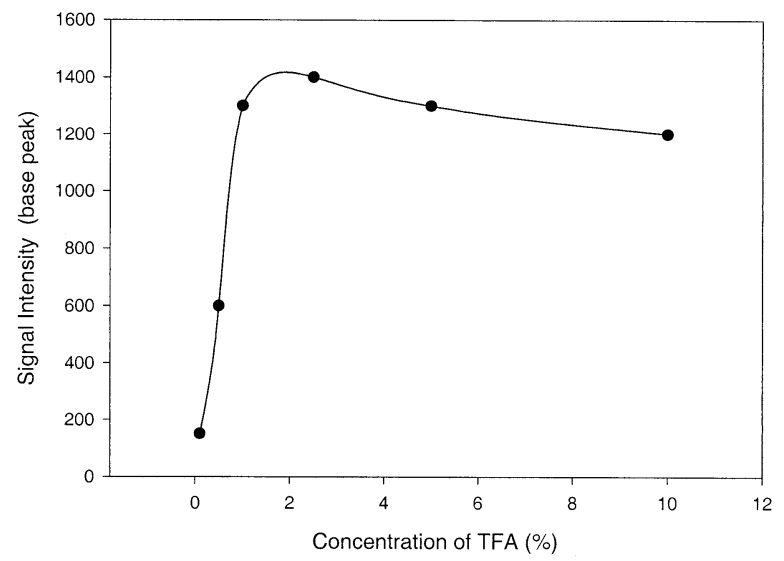

Figure 5. The graph of base peak signal intensity versus percent TFA concentration on the MALDA spectrum of E. coli in CHCA. (a)

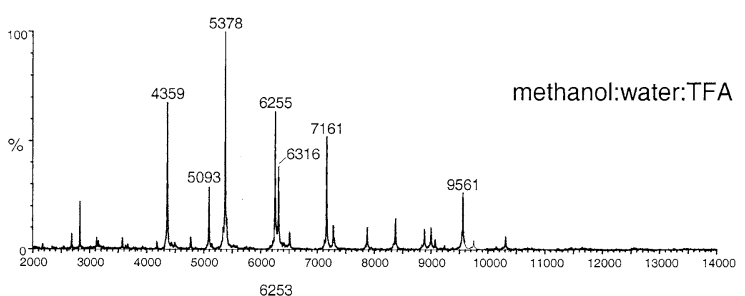

(b)

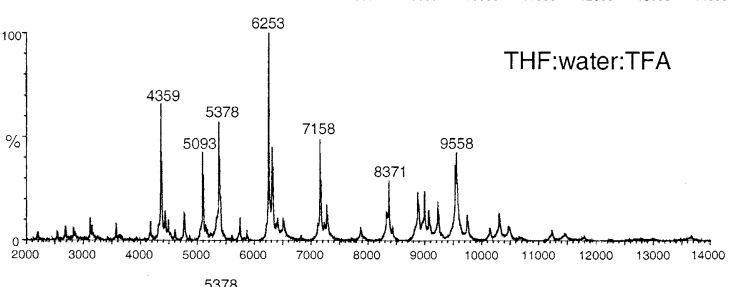

(c)

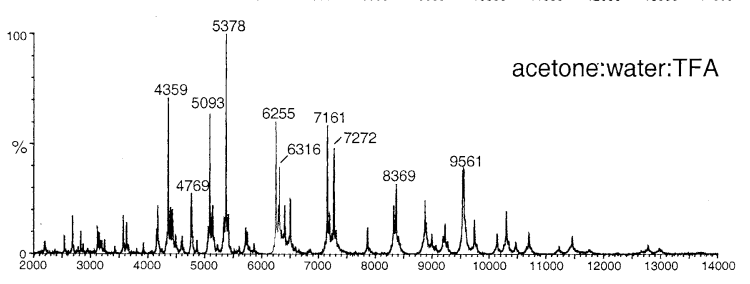

(d)

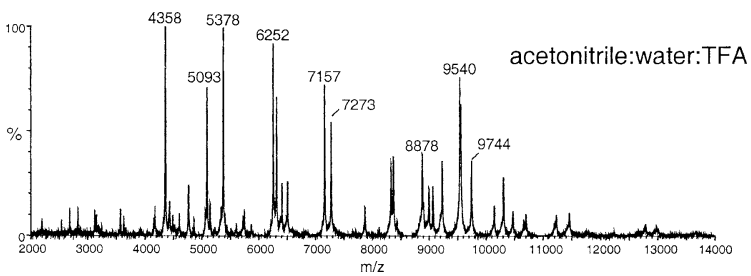

Figure 6. The effect of various matrix solvents on the number of protein peaks observed in the MALDI spectrum of E. coli. All solvents are a $50 / 50$ mix with $2.5 \%$ TFA.

$6 a$ and $b)$. THF appeared to be slightly better for the detection of proteins above $10 \mathrm{Kda}$. The spectra observed when using acetone or acetonitrile as the matrix solvent showed a lot of similarity (Figure $6 c$ and d). Both the number and intensity of low and higher mass proteins were better than with THF or methanol. However, the use of acetonitrile solvent consistently provided a better bacterial fingerprint in the 3 to $14 \mathrm{kDa}$ mass range than did acetone. These results may be attributed to the ability of aprotic solvents in the presence of acid to lyse cells resulting in the release of proteins.

Surfactant in the matrix. Our intent in developing a general and rapid MALDI TOF method for the analysis of whole bacteria cells was to obtain spectra that provide not only a good bacterial fingerprint in the 3 to 14 $\mathrm{kDa}$ mass range but also display good signal for the higher mass proteins present. We hoped that the higher energy associated with a Nd:Yag laser would enhance both the detection of higher mass proteins solubilized during lysis and those desorbed from the cell surface. Unfortunately, relatively few proteins greater than 20 $\mathrm{kDa}$ were detected and for those that were, the signal intensity was low. This was generally true under all the conditions previously discussed. This may be attributed to poor solubility of the higher mass proteins in the 

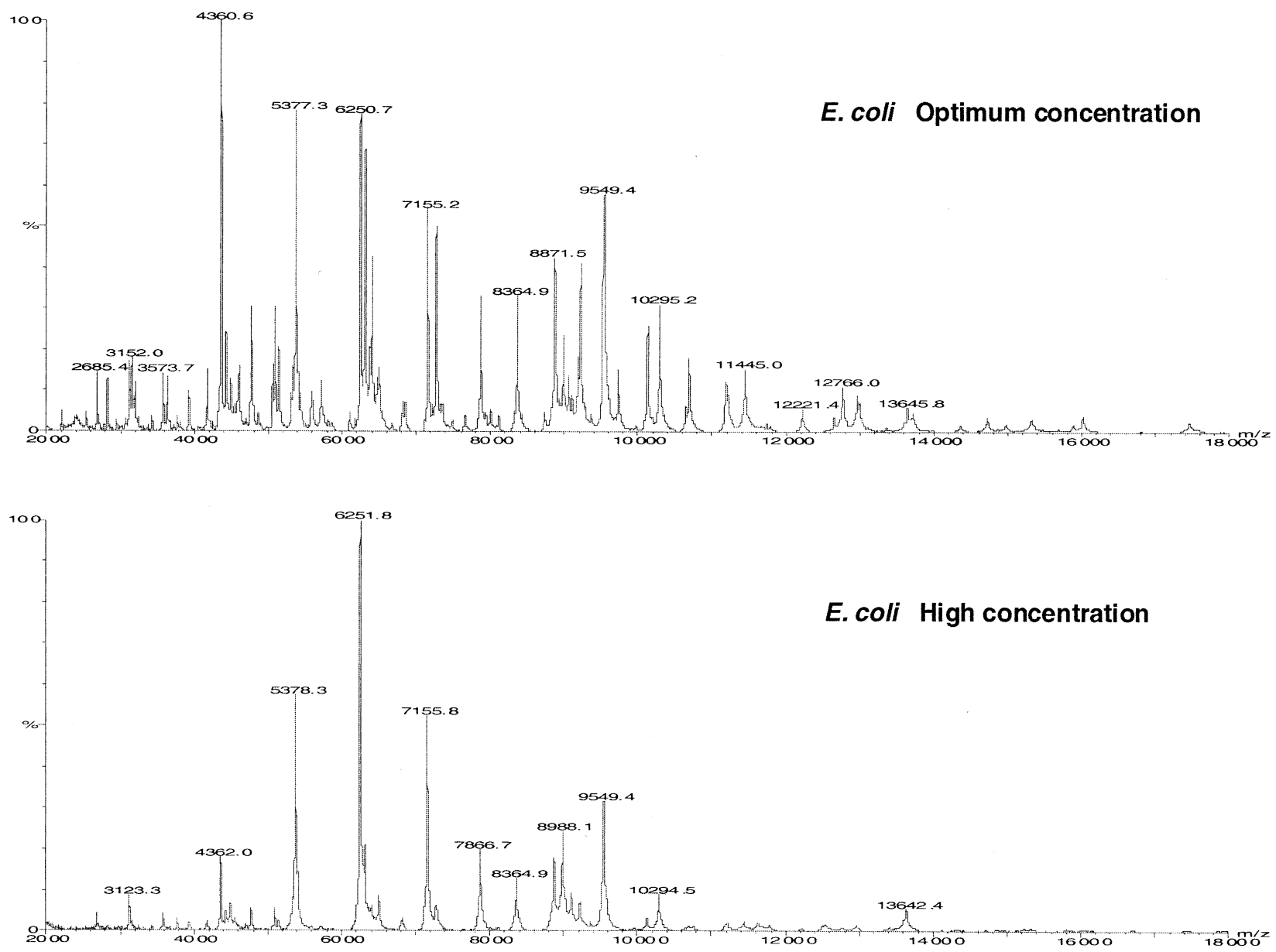

Figure 7. The effect of bacterial cell concentration on the MALDI spectrum. Serial dilutions were performed to find the optimum concentration for analysis.

matrix or instrument bias towards low masses. To investigate solubility effects and to increase the solubility of higher molecular weight proteins, we evaluated the effect of adding various surfactants to the CHCA matrix for sterilized E. coli and Listeria cells. The surfactants, Triton X100 and Tween 20R, were added to the matrix solvent mix at $0.1,0.5$, and $1.0 \%$ prior to mixing with the cell suspensions. The matrix and sample were then mixed and allowed to crystallize on the plate using the dried-droplet method. No significant enhancement of high mass proteins was observed using either of the surfactants and failure to observe high mass proteins was attributed to instrument performance.

\section{Bacterial Cell Concentration}

These studies were based on initial culture solutions having concentrations on the order of $1.0 \mathrm{OD}_{610}$, however no systematic study of the actual number of cells on the MALDI target were carried out. While careful attention was paid to the number of bacteria used in each experiment, significant variability of the spectra can be attributed to small variations in the number of cells on the MALDI target. Careful measurement and deposition of cells on the MALDI target is a tedious and time-consuming process, and despite careful control measures can result in poor reproducibility of the spectra. Because of these limitations and their obvious effect on spectral quality, we sought a faster, more reliable method of depositing an appropriate amount of bacteria on the MALDI target. By preparing three serial dilutions of bacteria with matrix, at concentrations of 1:1, 1:3, and 1:5 (bacteria:matrix), we found one combination always resulted in a high quality spectrum for the bacteria being analyzed (Figure 7). While serial dilutions of the bacteria with matrix allow for a fast and reproducible method for obtaining a high quality spectrum, the technique requires significant operator/interpreter decision-making and therefore does not currently lend itself to automated techniques. However, a more thorough description of the output of this process and integration into software algorithms would allow for automation.

\section{Instrumental Conditions}

Acceleration voltage. Changes in the accelerating voltage were examined to determine the optimum voltage 
for the analysis of whole cells sterilized using 70\% ethanol. Listeria innocua in CHCA was analyzed at 30, 25, 20, $15 \mathrm{kV}$ using the Nd:Yag laser. The maximum accelerating voltage of our instrument is $30 \mathrm{kV}$. The mass spectra at 30 and $25 \mathrm{kV}$ were essentially the same, exhibiting good resolution and a number of intense peaks between 2 and $12 \mathrm{kDa}$. At 20 and $15 \mathrm{kV}$ the peaks began to broaden, resulting in a significant drop in resolution as well as decrease in overall signal (data not shown). The optimum accelerating voltage was determined to be $25 \mathrm{kV}$.

$\mathrm{N}_{2}$ and Nd:Yag lasers. The Micromass TofSpec E in our laboratory was equipped with both the Nd:Yag and nitrogen laser. For the analysis of bacteria and in particular "whole cells", we were interested in determining if the higher energy density of the Nd:Yag laser would result in the detection of a greater number of peaks as well as in the detection of proteins of higher mass than that observed with the nitrogen laser. Both lasers were used to analyze the same spots of E. coli and Listeria grown from tryptic soy broth and agar plates. Under optimum conditions, no discernable difference in spectral quality was observed between the two types of laser. However, it was generally easier to obtain good spectra with the Nd:Yag laser than with the nitrogen laser. While the nitrogen laser often showed "hot spots" on the target, the Nd:Yag laser yielded good spectra without the need to search for a good spot on the target. Additionally, the Nd:Yag laser generally provided greater sensitivity and improved signal-to-noise ratios than did the nitrogen laser.

\section{Conclusions}

A systematic set of changes in experimental parameters was used to identify a set of conditions that gave optimized and reproducible MALDI TOF mass spectra from whole bacteria. The parameter set emphasized the use of practical bacterial sterilization solutions and other conditions consistent with minimum cost of complexity and maximum throughput. The final optimized protocol consisted of sterilization in $70 \%$ ethanol, a CHCA matrix in 50:50 acetonitrile:water with 2.5\% TFA, combined with serial dilutions of the bacterial and a Nd:Yag laser for ionization.

Considerable controversy exists regarding the physical condition of the bacterial cells prior to MALDI analysis. Electron microscopy has been used to show that whole bacterial cells are present on the MALDI target following complete sample preparation [24] while other citations indicate that $E$. coli and gram negative bacteria are readily lysed by the MALDI matrix solution $[11,25]$. Proteins observed by MALDI MS analysis are similar to those observed by LC/MS analysis of bacterial cell extracts [26], and thus, indicate that proteins are simply extracted from the cells during the addition of matrix, acid, and organic solvent. For this reason, matrix solutions have pronounced effects on the number and intensity of observed proteins. Therefore, the term "whole cell" MALDI best describes the condition of the cells applied to the MALDI target, but probably does not accurately reflect the final environment of the proteins prior to desorption/ionization. While some intact cells may be present, observed MALDI signals are most likely from proteins extracted from the cells and co-crystallized with the matrix.

Given the variables encountered with "whole cell" MALDI and the variation between instruments and manufacturers, it is extremely unlikely that spectra of bacteria obtained using this method will be directly comparable in all laboratories. Although many of the same masses will be observed, both intensity and masses, (particularly at higher masses), are likely to vary considerably. Combined with the poor mass accuracy of MALDI $( \pm 5 a \mu$ at 10,000$)$ and the inability of commercial search algorithms to factor mass resolution into the comparison, inter-laboratory comparison of the data is difficult. Therefore, in order to build a searchable library of MALDI spectra of bacteria it may be necessary to build data bases that are matched to a particular laboratory or instrument. For the purpose of species level identification this may not be necessary. However, for the detection of small differences at the strain level, it will be a requirement.

\section{References}

1. Cain, T. C.; Lubman, D. M.; Webber, W. J., Jr. Differentiation of Bacteria Using Protein Profiles from MALDI TOF MS. Rapid Commun. Mass Spectrom. 1994, 8, 1026-1030.

2. Holland, R. D.; Wilkes, J. G.; Rafii, F.; Sutherland, J. B.; Persons, C. C.; Voorhees, K. J.; Lay, J. O., Jr. Rapid Identification of Intact Whole Bacteria Based on Spectral Patterns Using Matrix-Assisted Laser Desorption/Ionization with Time-ofFlight Mass Spectrometry. Rapid Commun. Mass Spectrom. 1996, 10, 1227-1232.

3. Krishnamurthy, T.; Ross, P. L. Rapid Identification of Bacteria by Direct Matrix-Assisted Laser Desorption/Ionization Mass Spectrometric Analysis of Whole Cells. Rapid Commun. Mass Spectrom. 1996, 10, 1992-1996.

4. Claydon, M. A.; Davey, S. N.; Edwards-Jones, V.; Gordon, D. B. The Rapid Identification of Intact Microorganisms Using Mass Spectrometry. Nat. Biotechnol. 1996, 14, 1584-1586.

5. Arnold, R. J.; Reilly, J. P. Observation of Escherichia coli Ribosomal Proteins and Their Posttranslational Modifications by Mass Spectrometry. Anal. Biochem. 1999, 269, 105-112.

6. Lay, J. O., Jr. MALDI-TOF Mass Spectrometry and Bacterial Taxonomy. Trends Anal. Chem. 2000, 18, 507-516.

7. Mead, P. S.; Slutsker, L.; Dietz, V.; McCaig, L. F.; Bresee, J. S.; Shapiro, C.; Griffin, P. M.; Tauxe, R. V. Food-Related Illness and Death in the United States. Emerg. Infect. Dis. 1999, 5, 607-625.

8. Holland, R. D.; Rafii, F.; Heinze, T. M.; Sutherland, J. B.; Voorhees, K. J.; Lay, J. O., Jr. Matrix-Assisted Laser Desorption/Ionization Time-of-Flight Mass Spectrometric Detection of Bacterial Biomarker Proteins Isolated from Contaminated Water, Lettuce, and Cotton. Rapid Commun. Mass Spectrom. 2000, 14, 911-917.

9. Holland, R. D.; Duffy, C. R.; Rafii, F.; Sutherland, J. B.; Heinze, T. M.; Holder, C. L.; Voorhees, K. J.; Lay, J. O., Jr. Identification 
of Bacterial Proteins Observed in MALDI TOF Mass Spectra from Whole Cells. Anal. Chem. 1999, 71, 3226-3230.

10. Demirev, P. A.; Ho, Y. P.; Ryzhov, V.; Fenselau, C. Microorganism Identification by Mass Spectrometry and Protein Database Searches. Anal. Chem. 1999, 71, 2732-2738.

11. Fenselau, C.; Demirev, P. A. Characterization of Intact Microorganisms by MALDI Mass Spectrometry. Mass Spectrom. Rev. 2001, 20, 157-171.

12. Wang, Z.; Dunlop, K.; Long, S. R.; Li, L. Mass Spectrometric Methods for Generation of Protein Mass Database Used for Bacterial Identification. Anal. Chem. 2002, 74, 3174-3182.

13. Domin, M. A.; Welham, K. J.; Ashton, D. S. The Effect of Solvent and Matrix Combinations on the Analysis of Bacteria by Matrix-Assisted Laser Desorption/Ionization Time-ofFlight Mass Spectrometry. Rapid Commun. Mass Spectrom. 1999, 13, 222-226.

14. Saenz, A. J.; Petersen, C. E.; Valentine, N. B.; Gantt, S. L.; Jarman, K. H.; Kingsley, M. T.; Wahl, K. L. Reproducibility of Matrix-Assisted Laser Desorption/Ionization Time-of- Flight Mass Spectrometry for Replicate Bacterial Culture Analysis. Rapid Commun. Mass Spectrom. 1999, 13, 1580-1585.

15. Lay, J. O., Jr.; Holland, R. D. Rapid Identification of Bacteria Based on Spectral Patterns Using Matrix Assisted Laser Desorption Ionization (MALDI) Time-of-Flight (TOF) Mass Spectrometry. Methods Mol. Biol. 2000, 146, 461-488.

16. Lay, J. O., Jr. MALDI-TOF Mass Spectrometry of Bacteria. Mass Spectrom. Rev. 2001, 20, 172-194.

17. Dare, D. J.; Bright, J. J.; Sutton, H. E.; Edwards-Jones, V.; Keys, C. J.; Shah, H. N.; McKenna, T.; Lunt, M.; Wells, G. Rapid Identification of Bacteria Using Matrix Assisted Laser Desorption/Ionization Time-of-Flight Mass Spectrometry (MALDITOF-MS): A Database for Identification of Bacilli. Proceedings of the International Union of Microbiological Societies; Paris, France, July/August, 2002, http://www.micromass.co.uk/ litpdf/MMP237.pdf.
18. Wang, Z.; Russon, L.; Li, L.; Roser, D. C.; Long, S. R. Investigation of Spectral Reproducibility in Direct Analysis of Bacteria Proteins by Matrix-Assisted Laser Desorption/Ionization Ttime-of-Flight Mass Spectrometry. Rapid Commun. Mass Spectrom. 1998, 12, 456-464.

19. Madonna, A. J.; Basile, F.; Ferrer, I.; Meetani, M. A.; Rees, J. L.; Voorhees, K. J. On-Probe Sample Pretreatment for the Detection of Proteins Above $15 \mathrm{kDa}$ from Whole Cell Bacteria by Matrix-Assisted Laser Desorption/Ionization Time-of-Flight Mass Spectrometry. Rapid Commun. Mass Spectrom. 2000, 14, $2220-2229$

20. Lee, P. R. Irradiation to Prevent Foodborne Illness. J. Am. Med. Assoc. 1994, 272(4), 261.

21. Thayer, D. W.; Boyd, G. Elimination of Escherichia coli O157:H7 in Meats by Gamma Irradiation. Appl. Environ. Microbiol. 1993, 59(4), 1030-1034.

22. McDonnell, G.; Russell, A. D. Antiseptics and Disinfectants: Activity, Action, and Resistance. Clin. Microbiol. Rev. 1999, 12, 147-179.

23. Larson, E. L.; Morton, H. E. Alcohols in Disinfection, Sterilization, and Preservation. Lea and Febiger: Philadelphia, 1991; 4th ed.; 191-203.

24. Welham, K. J.; Domin, M. A.; Scannell, D. E.; Cohen, E.; Ashton, D. S. The Characterization of Microorganisms by Matrix-Assisted Laser Desorption Ionization Time-of-Flight Mass Spectrometry. Rapid Commun. Mass Spectrom. 1998, 12, 176-180.

25. Birmingham, J.; Demirev, P.; Ho, Y.-P.; Thomas, J.; Bryden, W.; Fenselau, C. Corona Plasma Discharge for Rapid Analysis of Microorganisms by Mass Spectrometry. Rapid Commun. Mass Spectrom. 1999, 13, 604-606.

26. Williams, T. L.; Leopold, P. A.; Musser, S. M. Automated Postprocessing of Electrospray LC/MS Data for Profiling Protein Expression in Bacteria. Anal. Chem. 2002, 74, 58075813. 